\title{
Some Exact Solutions of the Equations of Magne- tohydrodynamics when Both Self-Attraction and Magnetic Fields Are Present
}

\author{
G. C. McVittie \\ University of Illinois Observatory, Urbana, Illinois
}

\begin{abstract}
$\mathrm{T}$ HIS paper describes recent work on magnetohydrodynamics by M. H. Rogers and myself. It has seemed to us that the large-scale dynamics of cosmical gas clouds must take into account the fact that a gas is a compressible material, so that the hypothesis of a constant density must be avoided. Secondly, there must be present a pressure-gradient force. Thirdly, self-gravitation cannot be omitted. Fourthly, the motion should be adiabatic, in the first instance at least. To these conditions we have now also added the effects of the presence of magnetic fields, but have assumed that the conductivity of the material is infinite and that it is of constant permeability. We have neglected the effects of turbulence, of viscosity, and of heat conduction.
\end{abstract}

The fundamental equations of magnetohydrodynamics, with self-gravitation included, but with infinite conductivity assumed, are (in mks units)

$$
\begin{aligned}
& \text { electric vector, } \mathbf{E}=\mu \mathbf{H} \times \mathbf{u}, \\
& \text { current, } \mathbf{J}=\nabla \times \mathbf{H}, \\
& \text { magnetic vector, }(\partial \mathbf{H} / \partial t)+\nabla \times(\mathbf{H} \times \mathbf{u})=0 \\
& \text { and } \nabla \cdot \mathbf{H}=0 \text {, } \\
& \text { equations of motion, } \rho(\partial \mathbf{u} / \partial t)+\rho(\mathbf{u} \cdot \nabla) \mathbf{u}+\nabla p \\
& \qquad+\mu \mathbf{H} \times(\nabla \times \mathbf{H})=4 \pi G \rho \nabla \psi, \\
& \text { continuity equation, }(\partial \rho / \partial t)+\nabla \cdot(\rho \mathbf{u})=0, \\
& \text { Poisson's equation, } \nabla^{2} \psi=-\rho,
\end{aligned}
$$

and, without going here into discussion of the energy equation, the adiabatic condition

$$
(d / d t)\left(p \rho^{-\gamma}\right)=0 .
$$

In these equations, $u$ is the gas-velocity, $G$ the constant of gravitation, $\psi$ the "rationalized" gravitational-force potential, and $\gamma$ the ratio of the specific heats of the gas.

In seeking exact solutions we can consider situations possessing certain kinds of symmetry. Plane symmetry and cylindrical symmetry are the two simplest kinds in magnetohydrodynamics and these I shall concentrate on. As a second principle, it will be accepted that we can assign a functional form to one or more of the dependent variables. Usually the gravitational potential and/or the velocity of the gas are the ones best chosen for this purpose.
As a first illustration consider an idealized thin interstellar gas cloud envisaged as a slab of gas with plane-parallel faces whose width is small compared with its lateral extent. For mathematical simplicity we shall suppose that the slab is of infinite lateral extent. We ask whether such a slab can move as a whole perpendicularly to its plane faces, the width of the slab preserving the constant value $L$. The material is regarded as held together by its self-gravitation plus the effect of the magnetic field. The symmetry condition is that, if the $x$ axis be drawn normal to the faces of the slab, then the density, pressure, velocity, magnetic vector, and gravitational potential are to be functions of $x$ and $t$ alone. The functional form conditions are that the gravitational potential and the component of velocity parallel to the faces shall be arbitrary functions of

$$
X=x-\int v(t) d t
$$

where $v$ is also an arbitrary function of $t$. I shall not go through the process of solving (1) to (8) under these circumstances. It suffices to say that the $y$ and $z$ axes can be chosen so that $\mathbf{H}$ and $\mathbf{u}$ only have nonzero components $H_{x}, H_{y}$ and $u_{x}, u_{y}$, respectively, in the $x$ and $y$ directions, and that $\mathbf{J}$ only has one nonzero component, $J_{z}$. It turns out that the density is given in terms of an arbitrary function $f(X)$ by $\rho=d f / d X$; that $X=x-\alpha t$ where $\alpha$ is a nonzero constant; that $H_{x}$ is another nonzero constant $h$; that $\mathbf{u}$ is a linear function of $t$ and so does not depend on position; and that $H_{y}$ and the pressure are determined as functions of $f$ to within arbitrary additive constants $k$ and $\Pi$, respectively. Finally, it follows that the current $J_{z}$ is proportional to the density $\rho$.

To introduce precision into our solutions boundary conditions must be imposed. The slab is presumed to be a recognizable independent entity and since it consists of a compressible gas, we can define its plane faces by the requirement that the pressure and density shall vanish at these faces and that outside the slab there shall be a vacuum. This can be secured by making $d f / d X$ vanish at $X=0$ and $X=L$ and also by giving $I$ an appropriate value. But the electromagnetic boundary condition is more difficult. I believe that some experts hold that, at the surface of separation of a gas cloud and a vacuum, the current should vanish. In our case, 
since the current and density are proportional, this condition is automatically satisfied. Other investigators hold that it is the Lorentz force that should vanish, a condition usually expressed by saying that $\mathbf{J}$ is proportional to $\mathbf{H}$. But $\mathbf{J}$ always vanishes at the boundary, whereas $\mathbf{H}$ does not since $h$ is nonzero. Thus the proportionality of $\mathbf{J}$ and $\mathbf{H}$ cannot hold there. The magnetic vector can be continued outside the faces by a constant field. If in addition the magnetic energy per unit volume in front of, and behind, the slab is to have the same value, it follows that $H_{y}$ has equal and opposite values at the two faces and this fixes the constant of integration $k$. Thus the passage of the slab changes the direction of the magnetic vector outside the slab but not its magnitude.

With these boundary conditions the solution is (only nonzero functions are listed)

$$
\left.\begin{array}{rl}
X & =x-\alpha t, \\
\rho & =d f(X) / d X, \\
u_{x} & =\alpha t, \quad u_{y}=n \alpha t, \\
p & =-\left(1+\frac{n^{2} \alpha^{2}}{4 \pi G} \frac{1}{\mu h^{2}}\right)\{2 \pi G f(0) f(L) \\
\left.+\alpha f(X)+2 \pi G f^{2}(X)\right\}, \\
f(0)+f(L)=-\alpha / 2 \pi G, \quad \\
H_{x}=h=\operatorname{constant}, \\
H_{y}=(n \alpha / \mu h)\{f(X)+\alpha / 2 \Pi G\}, \\
J_{z}=n \alpha \rho / \mu h,
\end{array}\right\}
$$

where $\alpha, n$ are arbitrary constants and the slab extends from $X=0$ to $X=L$ at any instant $t$. Clearly the function $f$ cannot be chosen entirely arbitrarily but must be selected so as to make the pressure positive. It can be shown that if $n$ and $h$ are both zero the solution reduces to that in which only gravitational selfattraction and the pressure-gradient force act. Thus the corresponding magnetohydrodynamic solution implies that there is an accelerated flow of gas within the slab parallel to its faces in addition to the accelerated motion perpendicular to the faces. An individual particle of gas thus proceeds with a side-wise motion in space, the supply of gas being kept up because the slab is of infinite extent. In nature, therefore, such a motion could be expected to be transient in contrast to what happens in the purely self-gravitational case.

To demonstrate the squeezing effect of the magnetic field, I have worked out the expression for $L$ when the density is pre-assigned to be

$$
\rho=4 \rho_{m} \frac{X(L-X)}{L^{2}},
$$

where $\rho_{m}$ is the central density. For given central temperature and density in the slab, let $L^{\prime}$ be the width in the purely gravitational case and $L$ the width with the magnetic field. Then

$$
L=L^{\prime} /\left(1+\frac{n^{2} \alpha^{2}}{4 \pi G} \frac{1}{\mu h^{2}}\right)^{\frac{1}{2}},
$$

and thus $L<L^{\prime}$, the contraction factor depending on the ratio of the square of the acceleration parallel to the faces to twice the magnetic energy per unit volume due to the component of the magnetic vector perpendicular to the faces.

Dr. Rogers has considered the radial motions of an infinitely long cylinder of gas - an idealized spiral arm of a galaxy. The Eulerian method is used and cylindrical symmetry is assumed, all dependent variables being functions of $r$ (distance from the axis) and of $t$. The functional form condition is now the assumption that the velocity (which is radial only) obeys the linear wave rule, viz.,

$$
u=(r / R) d R / d t,
$$

where $R$ is a function of $t$ to be determined. It can be interpreted as the radius of the outer boundary of the cylinder. The solution of Eqs. (1) to (8) is then as follows: dependent variables are functions of $\zeta=r / R$ and of $t$; the nonzero components of the magnetic vector are the transverse $\left(H_{\theta}\right)$ and the axial $\left(H_{z}\right)$ where

$$
H_{\theta}=\frac{P(\zeta)}{R}, \quad H_{z}=\frac{1}{R^{2}} \frac{Q(\zeta)}{\zeta},
$$

$P$ and $Q$ being arbitrary functions of $\zeta$; the pressure and density are

$$
\rho=\frac{1}{R^{2}} \frac{h^{\prime}(\zeta)}{\zeta}, \quad p=S(\zeta) \rho^{\gamma}, \quad\left(h^{\prime}=d h / d \zeta\right),
$$

where $h$ and $S$ are arbitrary functions of $\zeta$; and finally $d^{2} R / d t^{2}$, which is a function of $t$ alone, is shown to be equal to three terms, each of which consists of a power of $R$ multiplied by a function of $\zeta$. Each of these three functions of $\zeta$ must therefore be equal to a constant and thus three equations are obtained; one of which gives $S$, the other $P$, and the third $Q$ in terms of the arbitrary density function $h$. By writing $R=R_{0} \chi$ and suitably adjusting the three constants, the differential equation for $R$ can be written as

$$
\frac{d^{2} \chi}{d t^{2}}+\frac{\lambda^{2}}{\chi^{2 \gamma-1}}+\frac{\sigma^{2}}{2(\gamma-1)} \frac{1}{\chi}-\frac{\kappa^{2}}{2(2-\gamma)} \frac{1}{\chi^{3}}=0 .
$$

Rogers then considers oscillatory motions, i.e., those in which $d^{2} \chi / d t^{2}=0$ when $R=R_{0}$ or $\chi=1$, and in such cases $\lambda^{2}$ is determined in terms of $\sigma^{2}$ and $\kappa^{2}$ from Eq. (10). It can be proved that, if $H_{\theta}$ is to be real up to the axis of the cylinder, then

$$
\sigma^{2} /(\gamma-1)>4 \pi G \rho_{0},
$$


where $\rho_{0}$ is the central density when $R=R_{0}$. It also follows that simple harmonic oscillations of small amplitude are possible if

$$
\sigma^{2}+\kappa^{2}>0,
$$

as found by Chandrasekhar and Fermi in their treatment of the small radial motions of such a cylinder of gas. The current has a transverse component involving $Q$ and an axial component involving $P$, but both components are proportional to the density $\rho$.

The application of the boundary conditions follows the same pattern as in the previous problem if the outer surface of the cylinder is defined by the condition that the density and pressure should both vanish there. This automatically satisfies the boundary condition that the current should be zero on the surface. Assuming also that $\mathbf{H}$ shall vanish at the surface of the cylinder, Rogers' oscillatory solutions are given by

$$
\begin{gathered}
\zeta=r R^{-1}=r R_{0}^{-1} \chi^{-1}, \\
\frac{d^{2} \chi}{d t^{2}}+\frac{\sigma^{2}}{2(\gamma-1)}\left[\chi^{-1}-\chi^{-(2 \gamma-1)}\right] \\
-\frac{\kappa^{2}}{2(2-\gamma)}\left[\chi^{-3}-\chi^{-(2 \gamma-1)}\right]=0,
\end{gathered}
$$$$
\rho=R_{0}^{-2} h^{\prime}(\zeta) \zeta^{-1} \chi^{-2},
$$$$
p=\frac{1}{4 \pi}\left[\frac{\sigma^{2}}{\gamma-1}-\frac{\kappa^{2}}{2-\gamma}\right](M-m) \chi^{-2 \gamma},
$$$$
u=\frac{d R / d t}{R} r=\frac{d \chi / d t}{\chi} r,
$$$$
\mu H_{\theta}{ }^{2}=\chi^{-2}\left\{-\frac{4 \pi G}{R_{0}^{2}} \frac{h^{2}}{\zeta^{2}}+\frac{\sigma^{2}}{\gamma-1} \frac{\int_{0}^{\zeta} h^{\prime} \zeta^{2} d \zeta}{\zeta^{2}}\right\},
$$$$
\mu H_{z}^{2}=\frac{\kappa^{2}}{2-\gamma} \frac{M-m}{2 \pi} \chi^{-4} \text {, }
$$$$
\mu J_{\theta}=\frac{\kappa^{2} R_{0}}{2(2-\gamma)} \frac{\zeta}{H_{z}} \frac{\rho}{\chi^{3}},
$$$$
\mu J_{z}=\frac{\sigma^{2} R_{0}}{2(\gamma-1)}\left\{1-\frac{8 \pi G(\gamma-1)}{\sigma^{2} R_{0}^{2}} \frac{h}{\zeta^{2}}\right\} \frac{\zeta}{H_{\theta} \chi} \frac{\rho}{\chi}
$$

Here $m$ is the mass within radius $r$ of unit length of the cylinder and $M$ the corresponding mass to the outer boundary. The vanishing of $H_{\theta}$ at the boundary places some slight restrictions on the function $h(\zeta)$, i.e., on the permissible internal density distributions. If $H_{z}$ is to be real, $\kappa^{2}$ must be positive; and for a positive pressure inside the cylinder we must have

$$
\frac{\sigma^{2}}{\gamma-1}>\frac{\kappa^{2}}{2-\gamma} \text {. }
$$

This inequality can be interpreted by thinking of the transverse component of the magnetic vector as squeezing the material of the cylinder together while the axial component tends to blow it apart. Now $\sigma^{2}$ is the constant that survives when the magnetic field is absent and self-gravitation is alone available to prevent the cylinder from flying apart. Thus the inequality (12) ensures that the disruptive effect of the axial component of the magnetic vector shall not be so great that only a state of internal tension could keep the cylinder together.

The solutions for both plane and cylindrical symmetry leave the internal density distribution unspecified except that the density must vanish at the boundaries. Thus they provide classes of exact solutions wherein no approximations have been made. They serve to emphasize the following points. (1) Compressibility is important: a gas can have an edge at which the density is zero without thereby having a zero density everywhere. This throws light on the applicability of electromagnetic boundary conditions. (2) It is rash to try and guess what the effect of the magnetic field will be by considering the situation when the field is absent. Examples are: the sidewise motion in the plane slab problem and the wide variety of radial motions in the cylindrical case according as gravitation, the transverse magnetic field or the axial field plays the dominant role. (3) The importance of similarity solutions in gas dynamics. In both problems the spacedependence enters essentially through the similarity variables $x / t$ or $r / R$. Rogers and I have conjectured that linear-wave flows of this kind must play a very fundamental role in gas dynamics but why this should be so, we have not yet been able to show. I can only end by expressing my conviction that these and other problems will be solved as more classes of exact solutions in all branches of gas dynamics are discovered.

\section{DISCUSSION}

R. LANDSHOFF, Lockheed Aircraft Corporation, Sunnyvale, California: I wonder to what extent it is legitimate to write the energy equation in the form that the entropy is constant. We do not have Joule heat because the conductivity is infinite, but we do have magnetic energies and Poynting vectors. Is it legitimate to simply write $p \propto \rho^{\gamma}$ ?

G. C. MCVITTIE, University of Illinois, Urbana, Illinois: No, not without going through the arguments 
and I said specifically that I would not go into them because they are rather complicated. The pressure is not simply proportional to $\rho^{\gamma}$ as you noticed. It is the ratio "following the motion" of $P^{-\gamma}$ that is constant so that one can have an arbitrary function of zeta coming in.

R. LANDSHOFF: That takes care of shocks, but what takes care of the magnetic energy?

G. C. MCVitTIE : Various proofs have been given that the adiabatic equation is a good enough approximation. In fact, I merely followed Friedrich's work on magnetohydrodynamics in this matter.

E. SchatzMan, Institut d'Astrophysique, Paris, France: I would like to draw the attention of $\mathrm{Dr}$. McVittie to another kind of nonlinear hydrodynamics. In the case of the slab of interstellar matter, the temperature inside the slab is not a constant because it's cooling. Then you have an equation of cooling which could be written $(d / d t)\left(\frac{1}{3} k T_{H} n_{H}\right)=-\gamma n_{H}^{2}$. If you add this to the equations of motion, you have another kind of nonlinear hydrodynamics which leads to a new eigenvalue problem.
G. K. BATCHELOR, Trinity College, Cambridge, England: Does Schlüter feel a need to comment on the matter of the boundary conditions?

A. SCHLÜTER, Max Planck Institut für Physik, Göttingen, Germany: The comment I should like to make about the boundary conditions is that one can assume either a slab of matter in vacuum, or one in a highly rarefied but conducting gas. In the first case, the normal component of the electric current has to vanish on the surface and one has to solve the usual Maxwell equations in the outer region. In the second case, current may flow across the interface between the dense and the rarified gas and, for the latter, one has to solve the equations for a free-force magnetic field.

G. C. MCVITTIE : Then you would not object if there was a vacuum outside with $\mathbf{J}$ equal to zero on the boundary?

A. SCHLÜTER : No, certainly not.

D. L. LAYZER, Harvard College Observatory, Cambridge, Massachusetts: I think your boundary conditions are determined by the equations inside the media and Maxwell's equations outside, and I believe one must take the continuity of the electric field as a condition. 